\title{
Nonlinearity Analysis and Compensation of Measuring the Moisture Content of Green Sand Based on Capacitance Method
}

\author{
SHI Dequan \\ College of Material Science and Engineering \\ Harbin University of Science \& Technology \\ Harbin, P. R. China \\ shidquan2008@163.com
}

\author{
GAO Guili \\ College of Material Science and Engineering \\ Harbin University of Science \& Technology \\ Harbin, P. R. China \\ gao-guili@163.com
}

\begin{abstract}
The capacitance method is one of the commonly-used methods for measuring the moisture content of green sand. However, due to the influence of the plate structure, the contact resistance and the parasitic capacitance and so on, there is a big non-linear problem. On the basis of briefly introducing the principle of measuring the moisture content by capacitance method, the nonlinearity of capacitance moisture sensor is analyzed theoretically, and the higher-order harmonics are taken as main reasons. Then, the influencing factors causing the nonlinearity are discussed. Finally, In order to reduce the nonlinear influence, a method for the nonlinear compensation based on the least square is provided and the experiments are performed. The experimental results show that it has good linearity between the input and output of the sensor, and the error is less than $2.0 \%$ after nonlinear compensation.
\end{abstract}

Keywords- nonlinearity; capacitance method; green sand; moisture content

\section{INTRODUCTION}

Green sand casting is an important and main kind of casting method because of the simple process, low cost and high productivity [1]. The performance stability of green sand directly affects the quality of the castings and production costs. Therefore, the measurement and control of green sand quality has been an important research topic in the foundry industry. The moisture content is one of key components that determined the main performance of green sand. Currently, there are many methods for measuring the moisture content of green sand [2, 3], and the capacitance method has been widely used for online measurement [4]. So, many researches have been performed and some available results have been achieved. Generally, in these results an alternating electric field is applied to the green sand sample, and the moisture content can be calculated indirectly by measuring the voltage of the sample. However, due to the influence of the plate structure, contact resistance and parasitic capacitance [5], it is hard to keep the linear relationship between the capacitance and the voltage of the measured sample in the whole range. Therefore, this nonlinear relationship seriously reduces the measurement accuracy of capacitance method.

On the basis of introducing the principle of the capacitance method measuring the moisture content, the nonlinearity

This work was supported by the Foundation for University Key Teacher of Heilongjiang Province of China (1252G025). between the capacitance and the voltage is theoretically analyzed, and the responding factors of influencing the nonlinearity are given. Then, based on the based on the least square method, a method for reducing the nonlinearity of the capacitance moisture sensor is provided and the experimental validation is done. This provides a foundation for further improving the accuracy that the moisture content is measured by the capacitance method.

\section{Principle of Measuring Moisture Content of GREEN SAND By CAPACITANCE METHOD}

Green sand is a mixture composed of the quartz sand, clay, water, coal powder and other additives. Among these components, the dielectric constant of dry matters is 2-4 while the dielectric constant of water is about 80 [6]. Thus, the small change of the moisture content is bound to cause the corresponding change of the dielectric constant of green sand. The higher the moisture content is, the larger the relative dielectric constant is, and thus the greater the capacitance is. On the contrary, the other components have little influence on the dielectric constant. Therefore, by measuring the capacitance of green sand the moisture content can be indirectly got. This is the basic principle of the capacitance method measuring the moisture content of green sand.

The capacitance moisture sensors have different construes according to the different casting condition. Fig. 1 shows the structure of typical cylindrical capacitance moisture sensor. The green sand is stored in the cylindrical sand-hopper which is used for one electrode of the sensor, and anther electrode is inserted into green sand. After the capacitance between electrodes is measured, the moisture content can be calculated according to the achieved capacitance-moisture curve.

When the sensor is filled with green sand, the relationship between the capacitance and dielectric constant can be described as:

$$
C=\frac{2 \pi \varepsilon_{0} \varepsilon_{x} L}{\ln R / r}
$$

where, $\varepsilon_{0}$ is the dielectric constant of vacuum, $\varepsilon_{x}$ is the dielectric constant of the measured green sand, $L$ is the 
effective length between the two electrodes, $r$ is the radius of the inner electrode, and $R$ is the radius of the outer electrode.

Therefore, the change of the capacitance $C$ is proportional to the change of the dielectric constant of green sand $\varepsilon_{x}$ when the sensor structure is fixed. If the alternating electric field is applied, by measuring the voltage between the two electrodes, the capacitance and thus the moisture content of green sand can be obtained.

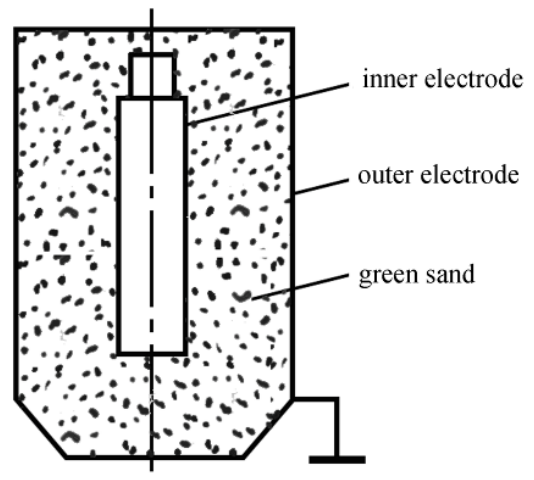

Figure 1 Schematic diagram of cylindrical capacitance sensor measuring moisture content of green sand

\section{NONLINEARITy ANALysis OF MEASURING MOISTURE CONTENT BY CAPACITANCE METHOD}

\section{A. Theoretical Analysis}

When the alternating electric field is applied to green sand, for the ideal sensor the voltage of green sand should be proportional to the current, and the volt-ampere characteristic wave is a straight line, and the corresponding impedance has nothing to do with the voltage. But in fact, due to the influence of many factors such as the drain conductance and the contact resistance on the capacitance, the relationship between the capacitance and the voltage is not strictly linear, and the impedance also decreases with the increase of the voltage. The causes of this nonlinearity will be analyzed as follows.

It is assumed that the instantaneous value of the applied voltage is $u$, then:

$$
C=C_{0}\left(1+a_{1} u+a_{2} u^{2}+a_{3} u^{3}+\cdots\right)
$$

where, $C_{0}$ is the capacitance of the sensor when the voltage is very low, and $a_{1}, a_{2}, a_{3}, \ldots$ are the constants.

If the instantaneous voltage is $-u$, then:

$$
C=C_{0}\left(1-a_{1} u+a_{2} u^{2}-a_{3} u^{3}+\cdots\right)
$$

Therefore, the relationship between the capacitance and the voltage can be written as:

$$
C=C_{0}\left(1+a_{2} u^{2}+a_{4} u^{4}+\cdots\right)
$$

If the alternating electric field is $u=U_{0} \sin \omega_{0} t$, the capacitance Q can be as follows:

$$
\begin{aligned}
Q & =C_{0}\left(u+a_{2} u^{3}+a_{4} u^{5}+\cdots\right) \\
& =C_{0}\left(U_{0} \sin \omega_{0} t+a_{2} U_{0}^{3} \sin ^{3} \omega_{0} t+a_{4} U_{0}^{5} \sin ^{5} \omega_{0} t+\cdots\right)
\end{aligned}
$$

where, $U_{0}$ and $\omega_{0}$ are the amplitude and frequency of the sinusoidal electric field, respectively.

The second order approximation is used, and the formula (5) can be simplified as:

$$
\begin{aligned}
Q=C_{0} & \left(U_{0}+\frac{3 a_{0} U_{0}^{3}}{4}+\frac{5 a_{4} U_{0}^{5}}{8}\right) \cdot \sin \omega_{0} t \\
& -C_{0}\left(\frac{a_{2} U_{0}^{3}}{4}+\frac{5 a_{4} U_{0}^{5}}{16}\right) \cdot \sin 3 \omega_{0} t \\
& +C_{0} \frac{a_{4} U_{0}^{5}}{16} \cdot \sin 5 \omega_{0} t
\end{aligned}
$$

Therefore, the current of green sand sample is:

$$
\begin{aligned}
i=\frac{d Q}{d t}= & C_{0} \omega_{0}\left(U_{0}+\frac{3 a_{0} U_{0}^{3}}{4}+\frac{5 a_{4} U_{0}^{5}}{8}\right) \cdot \cos \omega_{0} t \\
& -C_{0} \omega_{0}\left(\frac{3 a_{2} U_{0}^{3}}{4}+\frac{15 a_{4} U_{0}^{5}}{16}\right) \cdot \cos 3 \omega_{0} t \\
& +C_{0} \omega_{0} \frac{5 a_{4} U_{0}^{5}}{16} \cos 5 \omega_{0} t
\end{aligned}
$$

It indicates that when the sinusoidal excitation voltage $u=U_{0} \sin \omega t$ is applied, the third harmonic current $-C_{0} \omega_{0}\left(\frac{3 a_{2} U_{0}^{3}}{4}+\frac{15 a_{4} U_{0}^{5}}{16}\right) \cdot \cos 3 \omega_{0} t$ and fifth harmonic current $C_{0} \omega_{0} \frac{5 a_{4} U_{0}^{5}}{16} \cos 5 \omega_{0} t$ will generate as well besides the fundamental current $C_{0} \omega_{0}\left(U_{0}+\frac{3 a_{0} U_{0}^{3}}{4}+\frac{5 a_{4} U_{0}^{5}}{8}\right) \cdot \cos \omega_{0} t$. If the higher order approximation is adopted, the higher harmonics than five times can also be got. Therefore, the nonlinearity makes the current become non-sinusoidal, which will inevitably cause the error when the moisture content of green sand is measured.

\section{B. Influence Factors}

Although many factors can cause the nonlinearity of the capacitance moisture sensor, the following main factors will be discussed.

(1) The measured green sand sample. Green sand is a mixture containing water, and the water are polar molecules. Therefore, when the alternating electric field is applied, the dielectric polarization and energy loss occur and thus result in the nonlinearity of the capacitance moisture sensor. In addition, the other components having a polar molecule are involved in the dielectric polarization and energy loss, which will also cause the nonlinearity to some degree. 
(2) The structure and manufacturing process. The edge effect will make the design and calculation complex, reduce the sensitivity of the sensor, and thus cause the nonlinearity [7]. In addition, the nonlinearity is probably caused by the contact resistance between the electrode and the leading wire, the parasitic capacitance of wire, and residual magnetic field of metallic materials and so on. Therefore, shortening the leading wire length and using the shielded cable can effectively reduce the influence of parasitic capacitance.

(3) Leakage resistance. The capacitive reactance of the capacitance sensor is very high, particularly when the excitation frequency is low. However, when the total leakage resistance is close to the capacitive reactance, it is necessary to consider the impact of the leakage resistance which will depress the sensitivity and thereby cause the nonlinearity.

(4) Environmental factors. It includes the space radiation interference, ambient temperature and humidity and so on. The temperature will severely affect the sensor. On one hand, the relative position of the various components of the capacitance sensor will change with the temperature, resulting in an additional temperature error; On other hand, the temperature have a significant effect on the dielectric constant of water. Therefore, the change of temperature will inevitably lead to the change of the capacitance. This is the reason that the capacitance moisture sensor must be corrected by the temperature.

\section{COMPENSATION METHOD AND EXPERIMENTAL VALIDATION}

To compensate for the nonlinearity of capacitance moisture sensor, and make the input and output keep linear relation, the sensor is compensated by the inverse nonlinear correction method.

The basic principle of the inverse nonlinear correction method is shown in Fig.2. A nonlinear corrector is added in the sensor system. Assuming that the input signal is $x(t)$ and the output signal is $u(t)$, the nonlinear output relation is $u=f(x)$. After the nonlinear corrector is added, it is expected that the relation between the input and the output of system is linear, namely:

$$
y=x=f^{-1}(u)
$$

We can conclude that the nonlinear compensation can be achieved well if the nonlinear corrector has the output characteristics of the inverse function of the sensor.

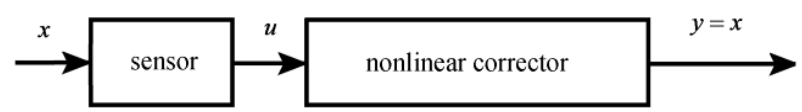

Figure 2 Schematic diagram of inverse nonlinear correction method

The least square method is used to establish the inverse nonlinear corrector [8], and the basic principle is as follows.
For known test data $\left(x_{i}, u_{i}\right)$, we try to find a function $x_{i}\left(u_{i}\right)=a_{0}+a_{1} u_{i}+a_{2} u_{i}^{2} \cdots+a_{m} u_{i}^{m}$ that makes the sum of square deviation $\sum_{i=1}^{n} \delta_{i}^{2}$ minimal, namely:

$$
\begin{gathered}
J\left(a_{0}, a_{1}, \cdots, a_{m}\right)=\min \sum_{i=1}^{n} \delta_{i}^{2}=\min \sum_{i=1}^{n}\left[x_{i}\left(u_{i}\right)-x_{i}\right]^{2} \\
=\min \sum_{i=1}^{n}\left\{\left[a_{0}+a_{1} u_{i}+a_{2} u_{i}^{2} \cdots+a_{m} u_{i}^{m}\right]-x_{i}\right\}^{2}
\end{gathered}
$$

where, $x_{i}\left(u_{i}\right)$ is the output of nonlinear corrector, $m$ is the polynomial order, $n$ is the number of data, and $a_{0}, a_{1}, \cdots, a_{m}$ are the coefficients that needed to be decided.

The selection of the polynomial order $m$ must be conducive to reduce the residual error sum of squares of each measurement point, and the F-test method is used to determine the number of the polynomial order. Generally, with the increase of the fitting polynomial order, the fluctuation of the fitting curve increases, and the curve gradually approaches the measuring points, and thus the corresponding residual error sum of squares reduces. At $m=n-1$ the fitting curve will accurately pass through all measurement points while the curve can not perfectly reflect the relationship between $x$ and ${ }^{y}$. Therefore we do not expect this situation appears. Usually, the selection of fitting polynomial order not only ensures the fitting curve is smooth, also embodies the intrinsic variation rule between the input and the output of the sensors. In addition, the floating-point arithmetic can be used to improve the accuracy of the fitting polynomial.

For simplicity, the fitting polynomial process is finished by Matlab, and the undetermined coefficients $a_{0}, a_{1}, \cdots, a_{m}$ can be automatically obtained.

Table I shows the data between the moisture content of green sand $x_{i}(\%)$ and output voltage of the sensor $u_{i}(\mathrm{~V})$ when the capacitance is detected by the bridge circuit. $u_{i}$ is taken as the input of the nonlinear corrector and $x_{i}$ is taken as the output of the nonlinear corrector, the fitting polynomial with seven order can be got according to the least square method.

$$
\begin{aligned}
x_{i}\left(u_{i}\right)= & -8.11769 \times 10^{-4}+0.68212 u_{i}+3.50186 u_{i}^{2} \\
& -9.84082 u_{i}^{3}+12.02872 u_{i}^{4}-6.92935 u_{i}^{5} \\
& +1.8794 u_{i}^{6}-0.1928 u_{i}^{7}
\end{aligned}
$$

TABLE I. DATA BETWEEN THE MOISTURE CONTENT OF GREEN SAND AND OUTPUT VOLTAGE OF THE SENSOR

\begin{tabular}{|c|c|c|c|c|c|c|c|c|}
\hline $\begin{array}{c}\boldsymbol{x}_{\boldsymbol{i}} \\
(\%)\end{array}$ & 0 & 0.51 & 0.98 & 1.43 & 2.34 & 2.66 & 3.14 & 3.54 \\
\hline $\begin{array}{c}\boldsymbol{u}_{\boldsymbol{i}} \\
(\mathbf{V})\end{array}$ & 0 & 0.47 & 0.87 & 1.21 & 1.60 & 1.82 & 2.11 & 2.31 \\
\hline $\begin{array}{c}\boldsymbol{x}_{\boldsymbol{i}} \\
(\boldsymbol{\%})\end{array}$ & 3.89 & 4.60 & 5.07 & 5.51 & 6.18 & 6.48 & 6.89 & 7.27 \\
\hline $\begin{array}{c}\boldsymbol{u}_{\boldsymbol{i}} \\
(\mathbf{V})\end{array}$ & 2.42 & 2.58 & 2.71 & 2.79 & 2.87 & 2.94 & 3.04 & 3.11 \\
\hline
\end{tabular}


After the nonlinear correction, the output characteristic wave of the sensor is shown in Fig.3. It can be seen that the curve is linear. The relative error curve of corrected sensor shown in Fig.4 indicates the relative error is less than 2.0\% in the whole range, which can meet the measurement requirements of the moisture content of green sand.

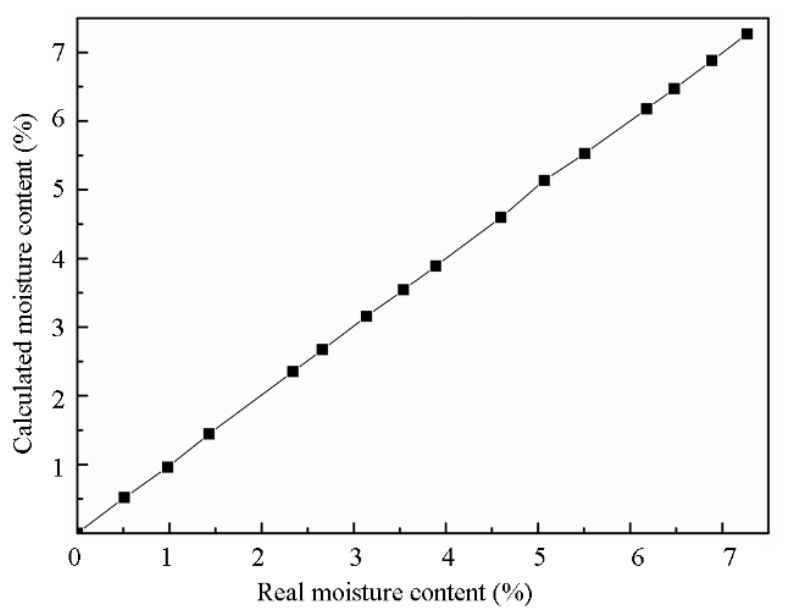

Figure 3 Output characteristic wave of the sensor after nonlinear correction

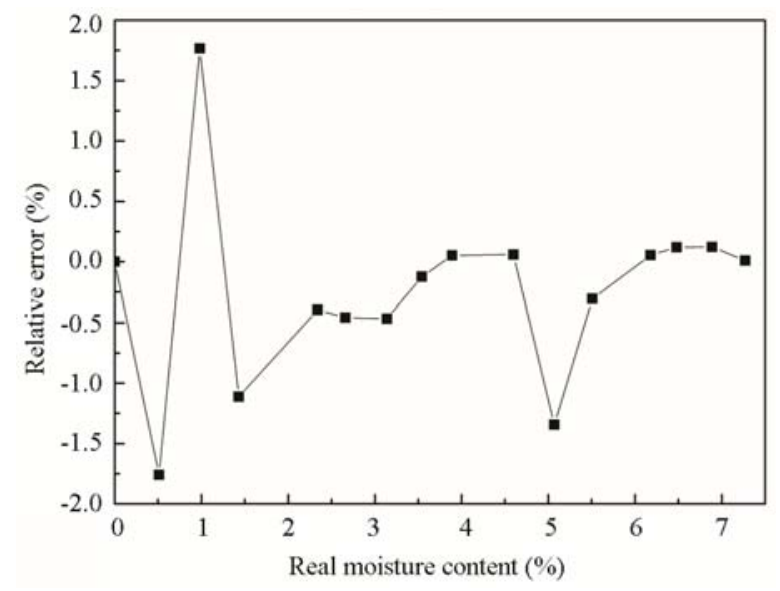

Figure 4 Relative error curve of corrected sensor

\section{CONCLUSIONS}

The principle of measuring the moisture content of green sand by the capacitance method is introduced. The moisture content is measured according to the fact that the dielectric constant of green sand has the single-valued relationship with the moisture but without the other components. Based on these, the reason of causing the nonlinearity is theoretically analyzed, and the higher order harmonics are main causes. The influencing factors, including the measured green sand sample, sensor structure and manufacturing process, leakage resistance and environmental factors are discussed. Then, a nonlinear compensation method based on the least squares is given to reduce the influence of the nonlinearity. The experiments show that the capacitance moisture sensor can keep a good linear relationship between the input and the output and the error is less than $2.0 \%$, which can meet the requirements of measuring the moisture content of green sand.

\section{REFERENCES}

[1] S. Kumar, P. S. Satsangi, and D. R. Prajapati, "Optimization of green sand casting process parameters of a foundry by using Taguchi's method,” International Journal of Advanced Manufacturing Technology, vol. 55, pp. 23-34, March 2011.

[2] H. R. Xiao, and W. W. Zhang, "Intelligent moisture controlling system for molding sand,” Advanced Materials Research, vol. 97-101, pp. 43094312, March 2010.

[3] Z. Li, Z. Huang, Y. Huang, and S. Deng, "Design of microwave moisture measuring instrument," Transactions of the Chinese Society of Agricultural Machinery, vol. 40, pp. 81-83, February 2009.

[4] S. Chen, J. Hu, and S. Zhu, "A review of development of mould sand moisture measuring and controlling instruments," Neural Networks, no. 3, pp. 47-51, March 2001.

[5] S. S. M. Nazirudeen, and B. Nagasivamuni, "Improving the quality of green sand castings to minimise the defects using artificial neural network,” Indian Foundry Journal, vol. 58, pp. 32-37, February 2012.

[6] D. P. Fernandez, Y. Mulev, and A. R. H. Goodwin, "A database for the static dielectric constant of water and steam,” Journal of Physical and Chemical Reference Data, vol. 24, pp. 33-70, December 2009.

[7] W. Wang, Y. Wen, J. Yu, and Z. Chen, "Impact of fringe effect on measuring accuracy of planar capacitive sensors," Sensor Letters, vol. 9, pp. 1458-1461, August 2011.

[8] G. Kemmer, and S. Keller, "Nonlinear least-squares data fitting in Excel spreadsheets,” Nature protocols, vol. 5, pp. 267-281, January 2010. 